\title{
Interactions of Peronospora tabacina with Roots of Nicotiana spp. in Gnotobiotic Associations
}

\author{
E. P. Heist, W. C. Nesmith, and C. L. Schardl
}

Department of Plant Pathology, University of Kentucky, Lexington 40546.

Accepted for publication 7 December 2001.

\begin{abstract}
Heist, E. P., Nesmith, W. C., and Schardl, C. L. 2002. Interactions of Peronospora tabacina with roots of Nicotiana spp. in gnotobiotic associations. Phytopathology 92:400-405.

Peronospora tabacina is an obligate plant pathogen that causes downy mildew disease on several species of Nicotiana, including $N$. tabacum (tobacco). The primary objective of this study was to use gnotobiotic associations to describe interactions between the pathogen and roots of either $N$. tabacum (cv. KY14) or N. repanda. We found that the pathogen was capable of moving systemically from shoots to roots of both host species and emerged from the root tissues as hyphae. We also demon-
\end{abstract}

ABSTRACT strated that root-associated hyphae were infectious on roots of nearby plants and resulted in new systemic infections. Following overnight darkness, sporulation of the pathogen was observed on infected roots exposed to air on both host species. We also found that within 2 months in culture, structures resembling resting stages of Peronospora tabacina were produced on hyphae emerging from roots of $N$. repanda but not $N$. tabacum. These findings appear relevant to both the epidemiology of the disease and to future studies of this and other downy mildew pathosystems.

Additional keywords: axenic, chlamydospore, oomycete, oospore, sporangia, tissue culture.
Peronospora tabacina D. B. Adam is an obligate plant pathogen that causes downy mildew, more commonly known as tobacco blue mold, on several plant species in the genus Nicotiana. Species of Nicotiana vary from immune to highly susceptible to blue mold (2). Included among the hosts regarded as highly susceptible is $N$. tabacum (tobacco), which is grown commercially in the United States and worldwide. Another host to the blue mold pathogen, characterized as tolerant to the disease, is $N$. repanda. This host is a wild relative of tobacco, native to the Southwestern United States and Mexico, and has been implicated as one of the primary overwintering hosts of Peronospora tabacina (21).

Historically, blue mold has been one of the most destructive diseases of tobacco, responsible for losses of hundreds of millions of dollars in revenue to tobacco growers $(9,12)$. Over the past 5 years, losses to blue mold have reached an estimated 350 million dollars in revenue to Kentucky burley growers. Therefore, losses to blue mold justify intensive investigation of the NicotianaPeronospora tabacina pathosystem.

Peronospora tabacina is an obligate biotroph requiring living host tissue to complete its life cycle $(8,22)$. Plant to plant spread occurs via asexual sporangiospores (sporangia) produced on the surface of infected leaves $(8,22)$. Infections resulting from colonization of the pathogen typically occur as either localized lesions on the leaves $(8,22)$ or can enter the vascular system and become systemic $(8,14)$. Conditions for sporulation of Peronospora tabacina on leaves of infected plants include relative humidity of $>95 \%$, temperatures in the range of 18 to $23^{\circ} \mathrm{C}$, and an overnight period of darkness (22).

Although there have been many investigations of foliar and systemic infections of Peronospora tabacina (14-16,19,20), little is known about potential interactions with host roots. Because tobacco plants are grown in situations that promote root-to-root contact, as in the modified hydroponic transplant production system (float

Corresponding author: E. P. Heist; E-mail address: epheis1@uky.edu

Publication no. P-2002-0212-01R

(C) 2002 The American Phytopathological Society system), it is imperative to verify whether the pathogen is capable of root-to-root transmission. This is important from both an epidemiological perspective and from the standpoint of designing the appropriate control strategies.

In a histological study, Milholland et al. (10) examined tissues of field-grown burley tobacco plants determined by symptoms to be systemically infected with Peronospora tabacina. They reported that the pathogen was confined primarily to the vascular tissue in the stem, but caused severe necrosis of all tissues in diseased roots. They also reported that infected stem and leaf tissues both contained mature oospores. Another study by Stanghellini et al. (18) on Plasmopara lactucae pv. radici, the root infecting downy mildew of lettuce, reported plant-to-plant transmission via rootinfecting motile zoosporangia. To our knowledge, this is the only report of a downy mildew pathogen capable of infecting host roots by propagules other than asexual sporangiospores as was reported by Novotelnova (13) for the sunflower downy mildew. Likewise, in previous experiments, we have also successfully initiated Peronospora tabacina infections by inoculating roots of tobacco plants with asexual sporangia.

Oospores of Peronospora tabacina occur in stem and leaf tissue of infected tobacco plants $(5,8,10,22)$. Since other oomycetes, like Pythium and Phytophthora, are capable of producing similar structures $(6,7)$, there is potential for any observed oospores produced by these opportunists to be mistaken for those of Peronospora tabacina. These observations are further complicated by the lack of evidence to support the hypothesis that oospores of Peronospora tabacina are capable of germination, thereby creating a situation in which proof of pathogenicity and matching these structures to their appropriate origin cannot be addressed. Furthermore, the manner by which oospores are detected in leaf and stem tissue (17) often requires killing the tissue. This might again complicate the issue of whether the observed oospores are actually from Peronospora tabacina and not from other closely related pathogens as mentioned above. Gnotobiotic associations of pathogen and host are needed to exclude the possibility that the oospores observed in association with infected host tissues are from Peronospora tabacina and not the product of secondary infection by closely related pathogens. 
Interactions of the blue mold pathogen with host roots have not been extensively studied. Furthermore, secondary spread of this pathogen has been traditionally viewed to occur via airborne sporangia $(1,3,22)$. Therefore, any secondary spread that was observed in previous studies was probably assumed to be from airborne sporangia without taking into account the potential for interactions occurring in the rhizosphere. These points illustrate the need for gnotobiotic associations of pathogen and host to enable us to examine roots of infected plants in a transparent medium (such as agar) and to more closely regulate sporulation of the pathogen to avoid confusion over whether secondary infections developed via airborne sporangia.

The primary objective of this study was to use the gnotobiotic associations we developed to determine whether Peronospora tabacina grows in association with roots of either $N$. tabacum or $N$. repanda and to determine if secondary spread occurs in these associations. In a second line of inquiry, we examined the pathogen for any novel morphology produced in association with roots of infected plants of both host species.

\section{MATERIALS AND METHODS}

Host material. Burley tobacco plants (N. tabacum cv. KY14) and the wild tobacco species $N$. repanda (provided by the Oxford Tobacco Research Station at North Carolina State University) were grown from surface-sterilized seed in petri plates $(100 \times 25 \mathrm{~mm})$ containing Murashige and Skoog (MS-) medium. Surface sterilization was performed by placing up to 400 seeds in 70- $\mu \mathrm{m}$ cell strainers (Becton Dickinson and Company, Franklin Lakes, NJ) and transferring to $70 \%$ ethanol for $3 \mathrm{~min}, 1.5 \%$ sodium hypochlorite solution for $30 \mathrm{~min}$, and followed by two sterile water rinses. Seeds were then transferred to MS- medium for germination. After germination, seedlings were transferred to fresh MS- medium, two plants per plate, for subsequent growth or inoculation. Plates were maintained in growth chambers (Model CU-36L5; Percival Scientific Inc., Boone, IA) at $23^{\circ} \mathrm{C}$ in $23-\mathrm{h}$ light/1-h dark. Light intensity was $66 \mu \mathrm{E} / \mathrm{m}^{2} / \mathrm{s}$.

Inoculum. Isolate KY79 of Peronospora tabacina $(14,15)$ was used for all inoculations in this study. It was collected from a tobacco field near Georgetown, KY, in 1979 and has been maintained at the University of Kentucky through frequent transfer into fresh tobacco plants. This isolate was experimentally determined to be sensitive to the fungicide metalaxyl.

Preparation of growth medium. MS- medium was modified from Murashige and Skoog (11) with $20 \mathrm{~g}$ of sucrose per liter and $9 \mathrm{~g}$ of phytagar (tissue culture grade, Gibco BRL, Grand Island, $\mathrm{NY}$ ) per liter. The $\mathrm{pH}$ was adjusted with $1 \mathrm{M} \mathrm{NaOH}$ between 5.7 and 5.9, and the medium was sterilized by autoclave. The antibiotics carbenicillin $(2.5 \mathrm{mg} / \mathrm{liter})$ and cefotaxime $(5 \mathrm{mg} / \mathrm{liter})$, (both from 1,000× stocks filter-sterilized with a $0.22 \mu \mathrm{m}$ Millipore filter; Millipore, Molsheim, France) were added to the sterile medium after it cooled to around $48^{\circ} \mathrm{C}$.

Maintaining gnotobiotic associations of pathogen and host in vitro. We obtained contaminant-free sporangia (spores) from cocultures of host and pathogen as described previously (4). Contaminant-free inoculum from cocultures was used to inoculate axenic host plants that were typically between 6 to 8 weeks old (due to differing growth rates between plants in vitro, some specimens were over 10 weeks old at the time of inoculation). Inoculum was maintained by inducing sporulation on infected plants and inoculating fresh axenic $N$. repanda plants with the spores every 1 to 2 months. Once infections were established on axenic $N$. repanda, sporulation could be induced to obtain sporangial suspensions of at least $5 \times 10^{3}$ sporangia per ml. Inoculations of each host species were made by applying 5 to 10 drops $(\approx 10$ to $20 \mu \mathrm{l}$ each) of inoculum to the surface of leaves with a hypodermic syringe equipped with a 25 gauge needle. Inoculated plants were transferred to darkness overnight to allow for spore germination and subsequent infection, then returned to 23 -h light and $23^{\circ} \mathrm{C}$ for subsequent growth. Symptom development was monitored visually, and roots were examined by microscopy for signs of pathogen egress.

Single spore inoculations. Single sporangia were obtained by evenly spreading spore suspensions $(0.5-\mathrm{ml}$ aliquots) of 500 spores per $\mathrm{ml}$ onto water agar in petri plates $(100 \times 15 \mathrm{~mm})$ and then, using a dissecting microscope, small areas of agar containing individual spores were picked up with a heat-pulled glass probe. These pieces of agar were placed spore side down in a small $(\approx 10 \mu \mathrm{l})$ drop of sterile water on leaves of individual axenic host plants ( $N$. tabacum and $N$. repanda) growing in MSmedium. Plants were transferred to overnight darkness for spore germination and subsequent infection. Because humidity in plates was adequate for spore germination, no additional moisture was added. Plants were then observed visually and by microscopy for symptom development. Following symptom development, the pathogen was induced to sporulate by placing plates in overnight darkness. Sporangia were collected from sporulating plants and used to single-spore inoculate a second round of axenic host plants to ensure infections were derived from a single sporangium of Peronospora tabacina. Infections resulting from the second round of single-spore-inoculated plants were monitored visually for symptom development and microscopically for root-associated pathogen growth.

Induction of sporulation from host roots. To observe sporulation from roots, plates containing newly infected plants were inverted to allow roots to grow gravitropically through the surface of the medium. When roots were visibly growing through the agar surface, plates were returned to upright position, and production of sporangiophores and sporangia (sporulation) was induced by moving plates to overnight darkness.

Root transmission experiments. To determine whether hyphae growing from roots of host plants were able to infect roots of nearby plants, axenic seedlings of both $N$. tabacum (cv. KY14) and $N$. repanda were plated onto MS- medium, two plants (designated 1 and 2, respectively) per plate and 10 plates (replicates) per host species. Once roots of plants 1 and 2 grew together, a sterile glass slide (dipped in $70 \%$ ethanol, flamed, and cooled) was inserted between them to separate the foliage. Thus, the only contact between the two plants was below the medium in the rhizosphere. Plant 1 was inoculated with 5- to $10-\mu$ droplets of a solution of $5 \times 10^{3}$ sporangia per $\mathrm{ml}$. Care was taken to ensure sporangia were placed only on the leaves. Plates were then moved into overnight darkness to allow for spore germination and infection. Plates were maintained in 23-h light to inhibit sporulation of the pathogen on each infected plant. Plants were monitored visually for symptom development on foliage and by microscopy for growth of the pathogen in association with host roots. Controls consisted of 10 plates per host species (two plants to a plate) in which the roots were kept from growing together by inserting a sterile glass slide down through the medium to the bottom of the plate, physically separating the root systems of plants 1 and 2 . Controls were inoculated similarly to test plants, one plant (1) per plate with 5- to $10-\mu$ l droplets of a solution of $5 \times 10^{3}$ sporangia per $\mathrm{ml}$. Controls were monitored for symptom development and rhizosphere activity similarly to test plants. Any Peronospora tabacina-inoculated plants not displaying symptoms at 10 days postinoculation were reinoculated. This process was repeated if necessary to ensure that all inoculated plants became infected. Root transmission experiments were performed five times for each host species with 10 replicates per experiment for both tests and controls.

Staining infected tissues for microscopic observation. Samples from infected tissues including stems, leaves, and roots were cleared and stained according to a procedure by Shipton and Brown (17). 


\section{RESULTS}

Peronospora tabacina was capable of moving systemically from the leaves of infected plants to the roots of both $N$. repanda and N. tabacum (cv. KY14). This was evident in both multiple and single-spore-inoculated plants as indicated by coenocytic (aseptate) hyphae emerging from the roots. In 'KY14' tobacco plants, the pathogen emerged from the roots as early as 6 days postinoculation. In the wild tobacco species $N$. repanda, the pathogen emerged from roots as early as 10 days postinoculation in a manner similar to that observed for infected ' $\mathrm{KY} 14$ ' tobacco (Fig. 1A). Emergence of hyphae from roots of plants that were single-spore-inoculated was delayed by an additional 3 to 5 days compared with plants inoculated with higher numbers of sporangia.

Initially, the pathogen only grew from root tips (first 2 to 3 weeks postinoculation), but eventually emerged from the root epidermis at various points in both host species. Two to three weeks following inoculation, hyphae became more abundant and extended farther into the medium (Fig. 1B). Over the course of the study, hyphae of the pathogen emerging from roots could easily be seen with the unaided eye and grew up to $3 \mathrm{~mm}$ from roots of 'KY14' plants and up to $10 \mathrm{~mm}$ from roots of $N$. repanda. Hyphal elongation from roots of 'KY14' plants continued for over 1 month and from roots of $N$. repanda for up to 6 months after inoculation.

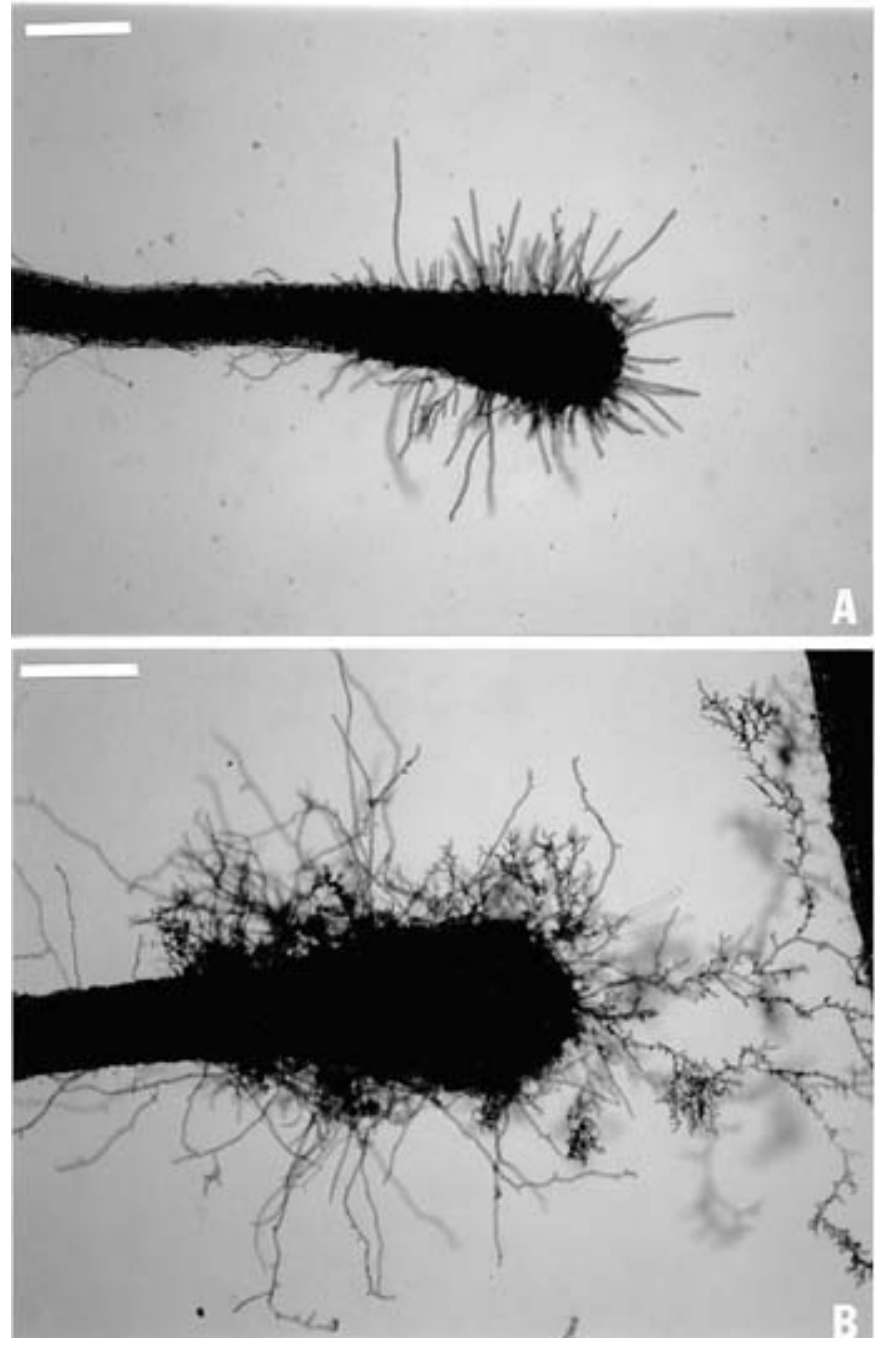

Fig. 1. Hyphae of Peronospora tabacina emerging from root of infected Nicotiana repanda plant at $\mathbf{A}, 14$ days and $\mathbf{B}, 3$ weeks following inoculation of foliage. Bars $=200 \mu \mathrm{m}$.
Foliar portions of 'KY14' tobacco and $N$. repanda became necrotic within 2 to 3 weeks and 2 months, respectively, following foliar inoculation if plants were not induced to sporulate. Induction of sporulation on infected host plants caused a comparatively rapid onset of necrosis. Necrosis was evident within 1 week following sporulation on 'KY14' tobacco plants and 3 weeks on $N$. repanda plants. However, pathogen outgrowth from the roots continued despite complete necrosis of foliar portions of host plants. Uninoculated controls did not become necrotic following overnight darkness regardless of host species.

Sporulation of the pathogen occurred on roots exposed to air following induction in overnight darkness (Fig. 2). Sporangiophores with asexual sporangia were typically observed to emerge from root tips but could also be seen at varying intervals along the root epidermis. Following induction, sporulation of the pathogen occurred on nonnecrotic portions of foliage of infected $N$. tabacum and $N$. repanda plants for up to 3 weeks and 3 months, respectively, following inoculation. Sporangia produced from roots and leaves were collected and used to complete Koch's postulates on susceptible host plants, verifying that the infections were indeed from Peronospora tabacina.

In our root transmission experiments, we demonstrated that hyphae emerging from roots of foliar inoculated plants ( 1 or vector plants) were pathogenic to roots of nearby uninoculated plants ( 2 or recipient plants) and resulted in new systemic infections. Transmission of pathogen to 2 plants via root-associated hyphae usually occurred within 1 month following foliar inoculation of 1 plants in both host species. Symptom development (chlorosis progressing from the base of the stem to the leaves) on 2 plants typically occurred within 1 week following observation of hyphal contact with roots. Results of root transmission experiments are summarized in Table 1. Once systemic symptoms (chlorosis and blistering progressing from the base of the stem to the leaves) were evident on 2 plants, sporulation was induced by placing in overnight darkness. Sporulation was commonly observed to cover up to $100 \%$ of the surface of these plants (both stems and leaves), indicating systemic infection. To verify that 2 plants in root transmission experiments had been infected by Peronospora tabacina, we collected sporangia following induction of sporulation and used them to complete Koch's postulates on susceptible host plants. In controls, in which root systems of the 2 plants were kept physically separated, infections occurred only on the inoculated plants (Table 1).

Interestingly, between 45 and 60 days postinoculation, structures resembling the sexual stages (oogonia, antheridia, and oospores) of Peronospora tabacina $(8,10)$ were observed in

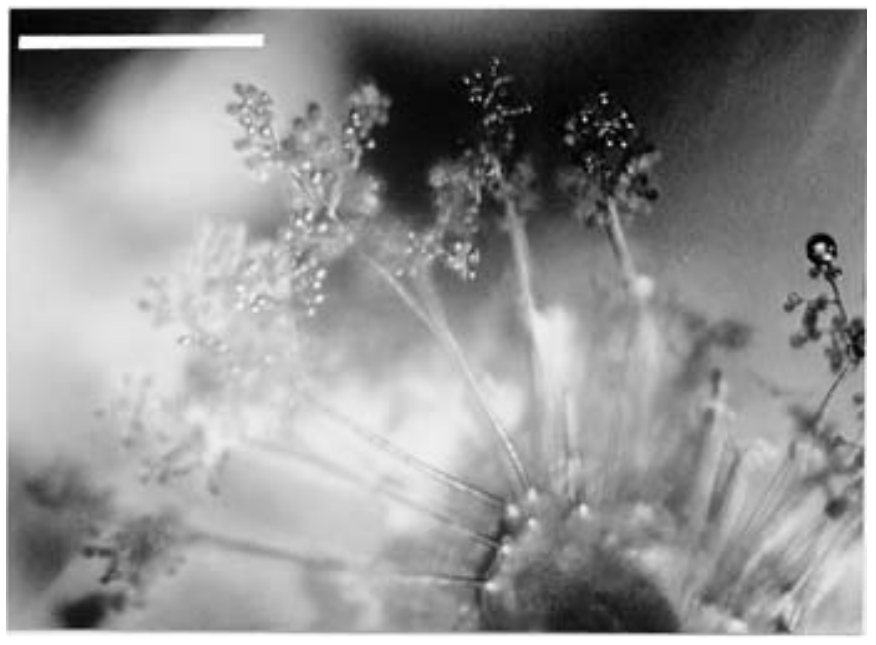

Fig. 2. Peronospora tabacina sporulating from roots of cv. KY14 tobacco. $\mathrm{Bar}=0.5 \mathrm{~mm}$ 
association with roots of infected $N$. repanda (Fig. 3A), but not 'KY14' tobacco. Several of the putative oospores were characterized by what appeared to be an oogonium with either single or multiple antheridia. These putative oospores and oogonia were only formed on hyphae external to roots of infected $N$. repanda and were not observed in any internal leaf, stem, or root tissue following staining and microscopic observation. Putative oospores and oogonia were continuously produced, beginning after the first month, over the course of 6 months in association with root systems of all infected $N$. repanda in this study. Putative oospores ranged from 24 to $56 \mu \mathrm{m}$ in diameter and averaged $38 \mu \mathrm{m}$ in diameter (average of 25 putative oospores). Germination of putative resting structures was not observed in this study. Furthermore, due to a lack of specimens and the need to further examine these structures in their undisturbed niche for any activity (e.g., germination or further development), experiments to determine if these structures are infectious were not performed.

Structures characteristic of other oomycetes were observed in association with roots of infected $N$. repanda plants. These included putative chlamydospores (Fig. 3B) and allantoid sporangialike structures (Fig. 4). Putative chlamydospores were formed either as terminal structures on hyphal tips or as hyphal invaginations occurring within the length of the hyphae. These structures had thick outer walls ( 1 to $2 \mu \mathrm{m}$ thick) and resembled chlamydospores produced by Phytophthora spp. (6). Putative chlamydospores ranged from 10 to $24 \mu \mathrm{m}$ in diameter and averaged $16 \mu \mathrm{m}$ in diameter (average of 25 putative chlamydospores). Structures resembling sporangia similar to those produced by Pythium spp. (7) were also found in association with infected roots of $N$. repanda. These structures were darkly pigmented and appeared to have a septum where they connected to the hypha. In Pythium spp., structures similar to the ones seen here (Fig. 4) are usually associated with zoosporangia (motile asexual spores) production (7). However, no zoosporangia were observed in association with these structures. It is likely that zoospore production, if a possibility here, would require flooding the substrate with water, which was not done in this study.

To address the possibility that the observed structures (potential oospores, chlamydospores, and sporangia) resulted from contamination by Phytophthora or Pythium spp., infections were generated on both host species using single spores of Peronospora tabacina and in every case resulted in production of the aforementioned structures. Furthermore, no such structures or pathogen hyphae were observed in association with any uninoculated control plants. In addition, representative isolates of both Pythium and Phytophthora spp. were placed on MS- medium and both grew actively (mycelium grew at least $3 \mathrm{~cm}$ from a small block of colonized agar) within 1 week. Therefore, it is unlikely that any structures observed in these associations were produced by contaminating microbes.

TABLE 1. Results of five root transmission experiments

\begin{tabular}{lccccc}
\hline & \multicolumn{4}{c}{ Root transmission $^{\mathrm{a}}$} \\
\cline { 2 - 3 } & \multicolumn{2}{c}{ Nicotiana repanda } & & \multicolumn{2}{c}{ Nicotiana tabacum $^{\text {Experiment }}$} \\
\cline { 2 - 3 } \cline { 5 - 6 } Test $^{\mathrm{b}}$ & Control $^{\mathrm{c}}$ & & Test $^{\mathrm{b}}$ & Control $^{\mathrm{c}}$ \\
\hline 1 & 10 & 0 & & 4 & 0 \\
2 & 10 & 0 & & 1 & 0 \\
3 & 10 & 0 & & 2 & 0 \\
4 & 10 & 0 & & 1 & 0 \\
5 & 10 & 0 & & 2 & 0 \\
\hline
\end{tabular}

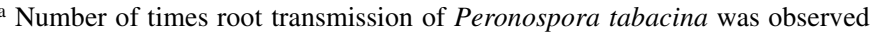
out of 10 replicates within each experiment for each plant species.

${ }^{b}$ Test plates contained two plants with the foliage physically separated prior to and after inoculation with Peronospora tabacina.

c Control plates contained two plants with the roots physically separated prior to and after inoculation with Peronospora tabacina.

\section{DISCUSSION}

Using gnotobiotic associations of host and pathogen, we demonstrated root transmission of Peronospora tabacina in both wild and commercial species of Nicotiana in vitro. The infectious agent was most likely hyphae that emerged from roots of infected plants. One important consideration regarding the root transmission experiments is how to exclude the possibility of pathogen transmission via airborne sporangia. To avoid sporulation of the pathogen, we maintained plants in 23-h light. As an added precaution, the plants in our root transmission experiments were physically separated by inserting a sterile glass slide between the foliage so the only contact between the two plants in each replicate was in the rhizosphere, below the agar surface. We also included controls that featured plants whose root systems were physically separated. No plant-to-plant transmission of Peronospora tabacina from the inoculated to the noninoculated plants was observed in these controls.

Although sporulation of the pathogen was inhibited in 23-h light, there was some abnormal sporangiophore and sporangia production on leaves of both test and control plants. These were similar to what was observed in our earlier studies with Peronospora tabacina on host callus (4). These abnormal sporangia apparently were not infectious under the conditions of our experiments as indicated by absence of transmission in controls where there was foliar contact with infected plants displaying abnormal sporangia.

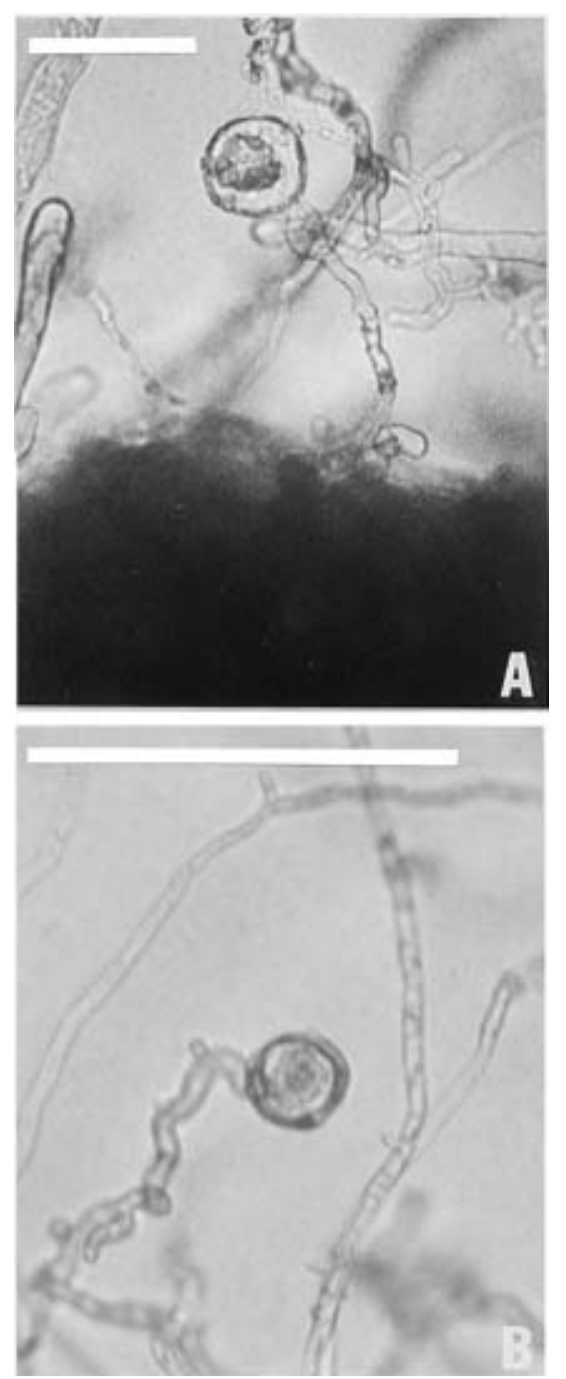

Fig. 3. A, Putative oospore and B, chlamydospore of Peronospora tabacina produced in association with roots of infected Nicotiana repanda plants at 45 days following inoculation of leaves. Bars $=50 \mu \mathrm{m}$. 
Root transmission typically occurred within the first month following inoculation of vector plants (1 plants) in experiments with both $N$. tabacum (cv. KY14) and $N$. repanda. Root transmission occurred in all replicates with $N$. repanda but in only 10 to $40 \%$ of 'KY14' tobacco plants (Table 1). One possible reason for lower transmission rates in the tobacco cultivar compared with the wild species was that the tobacco plants did not grow as well in this system as did $N$. repanda. Furthermore, root contact between the inoculated and uninoculated tobacco plants in the root transmission experiments was minimal. In addition, Peronospora tabacina hyphae growing from roots of 'KY14' tobacco plants were typically much shorter than hyphae growing from $N$. repanda roots $(3 \mathrm{~mm}$ compared with $10 \mathrm{~mm}$, respectively). However, despite the difficulties imposed by poor growth of the tobacco species in vitro, we repeatedly demonstrated root transmission. Therefore, root transmission may be a potential mode of secondary spread in tobacco seedling production systems which features much more root-to-root contact between neighboring plants than was typical in this study (E. P. Heist and W. C. Nesmith, unpublished data).

Infections were observed to progress from the leaves to the roots of all inoculated plants. To ensure that root infections had originated in the leaves and had not resulted from inadvertent placement of sporangia on the stems or roots, single spore inoculations were carried out. We found that the pathogen also grew from roots of all host plants inoculated on the leaves using single spores, indicating that the pathogen had indeed moved systemically from shoots to roots.

Another important question regarding our conclusion that hyphae acted as the infectious agent in the root transmission experiments was whether secondary spread occurred from the putative resting structures (putative oospores or chlamydospores). First, root transmission usually occurred prior to formation of putative resting structures (within 30 days compared with 45 to 60 days, respectively). Second, root transmission in tobacco plants was demonstrated, in which no putative resting structures were observed. Finally, putative resting structures produced in association with roots of infected $N$. repanda did not appear to germinate throughout the course of our experiments ( $>6$ months). Complex conditions may be required for germination of these structures and subsequent infection.

We observed what appeared to be sexual structures (oospores) of Peronospora tabacina produced on hyphae emerging from roots of $N$. repanda but not 'KY14' tobacco. These structures appeared to consist of an oogonium with either single (most common) or multiple antheridia. Unlike previous studies on infected

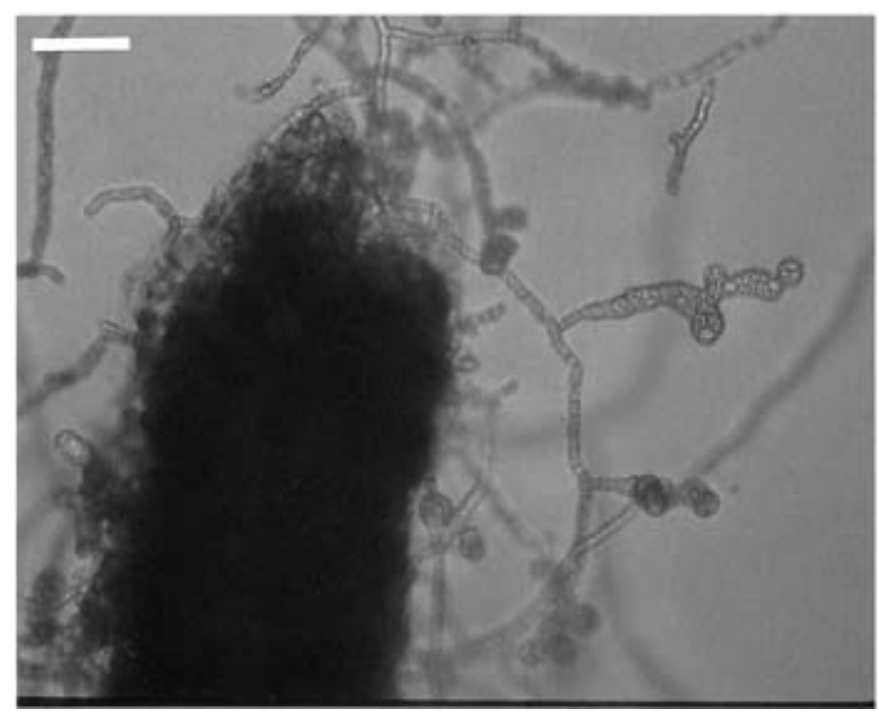

Fig. 4. Sporangia-like structures resembling those produced by Pythium spp. Bar $=30 \mu \mathrm{m}$. plants from field or greenhouse environments that may have included interactions of morphologically similar pathogens, this study was done using gnotobiotic associations of pathogen and host. Therefore, the observed oospore-like structures in this study must have been produced by Peronospora tabacina, whereas this could not be ensured for the previous reports. Also, there were several putative oogonia produced (no obvious antheridia attached). The sizes of these structures were consistent with the 20 to $60 \mu \mathrm{m}$ reported in the literature for oospores and oogonia of Peronospora tabacina (8). One major difference between the putative oospores seen here and those reported in the literature is that these were formed on hyphae external to the root, whereas those mentioned in the literature were always formed in either infected leaf, stem, or root tissue $(5,8,10)$. Whether the oosporelike structures observed actually resulted from sexual recombination or were merely oogonia with attached antheridia (without sexual recombination), produced as a result of the unique environmental conditions, remains unknown. Nevertheless, these observations appear relevant to future studies of this pathosystem due to the importance of oospores as a potential source of primary infection. Also, several different isolates of Peronospora tabacina need to be screened for their potential to produce mature oospores, both from the standpoint of identifying isolates with potential oosporeproducing capabilities and as a source of oospores for research.

Another important observation was that putative oospores were produced from hyphae emerging from roots of plants in which the infection was generated from a single spore. If the observed structures were indeed oospores this might suggest that the isolate of Peronospora tabacina used in this study is homothallic. However, to prove this will involve examining multiple isolates in both single and multiple spore infections and verifying whether these structures are actually a product of sexual recombination or just vestigial structures formed as a result of the unique environment.

Peronospora tabacina continued to grow from the roots of infected hosts many months following total necrosis of foliar portions of the plant. This finding raises the possibility that the roots may provide an excellent environment for prolonged propagation of the pathogen. It is also possible that host roots might be an effective overwintering source for the pathogen. Root infections may also be important for secondary spread in seedling production systems such as the float system, which offer young succulent host tissue as in our experiments. Much work is needed to address these and other hypotheses pertaining to the behavior of this pathogen in field and greenhouse environments.

Sporulation was observed to occur from roots of infected plants of both host species. These results might suggest that secondary spread of the disease also occurs from sporangia produced on the roots. If so, this might complicate control strategies used in seedling production systems that generally focus on foliar protection. These matters may be further complicated when metalaxyl-resistant strains of Peronospora tabacina are present or when nonsystemic fungicides are used.

Studies with regard to potential root transmission with other cultivars of tobacco and multiple isolates of Peronospora tabacina need to be carried out to determine if different cultivars are at higher risk for secondary spread via root transmission. If root transmission proves to be a reliable mode of secondary spread in seedling production systems or other nonaxenic situations involving infected plants, this may be something to consider when devising resistance strategies. Attempts to generate genetic resistance in plants or fungicide resistance have typically focused on ability to prevent spore germination and subsequent infection and colonization on leaves of host plants.

Although studies have been carried out in the past with respect to root transmission in other downy mildew systems $(13,18)$, to our knowledge, this is the first to investigate root-to-root transmission of Peronospora tabacina. Using gnotobiotic associations of the blue mold pathogen with host plants, we were able to look 
at the interactions differently than we have in the past, which has traditionally involved plants grown in soil. Use of the gnotobiotic system to study interactions between the pathogen and host roots has several advantages. First, it avoided contamination that might confound results. Also, because the growth substrate was agarbased, we had the added advantage of observing roots of hosts at all stages of plant growth and infection by the pathogen without using invasive measures.

It should be noted that the conditions under which these experiments were performed differ greatly from the various soil environments under which tobacco is grown in nature. In addition to the many different soil types (clay versus sandy), there are vast differences in the microbial communities that inhabit any given soil type. Microbial communities in the soil are influenced by complex factors such as soil $\mathrm{pH}$, available moisture, redox potential, and aggregation of soil particles. These and other factors would more than likely influence root transmission of Peronospora tabacina in commercial tobacco production systems, and should be examined in future studies. Therefore, to determine whether Peronospora tabacina is transmitted via roots of infected tobacco plants in nature requires repeating these experiments on different tobacco varieties grown in various soil types that differ both in their physical and chemical composition as well as in their microbial constituents. Experiments with varying environmental conditions should also be performed. We speculate that the most likely scenario for root transmission of Peronospora tabacina in production environments is the tobacco float production system, which features young succulent tobacco plants with intertwined root systems. In addition, the float system provides high humidity and moisture required for development of tobacco blue mold. Foreseeable problems associated with studying root transmission of Peronospora tabacina and formation of putative resting structures in association with tobacco plants grown in soil include (i) inability of an investigator to examine roots at all stages of infection, (ii) prevention of sporulation on plant tissues below the soil surface could not be ensured, making it difficult to assess whether hyphae emerging from the roots can cause infection, and (iii) resting structures produced external to the roots would be difficult, if not impossible, to find and remove from the soil for further study.

Using this whole plant gnotobiotic system, we have demonstrated that the pathogen can be transmitted from roots of infected plants to roots of nearby uninfected plants, probably via hyphae that emerge from the roots. We found what appeared to be resting structures (putative oospores or chlamydospores) formed in association with hyphae emerging from roots of infected $N$. repanda. These findings appear both relevant to future studies of this pathosystem from the standpoint of potential secondary spread in commercial tobacco production systems and to address questions pertaining to the life cycle of Peronospora tabacina in both wild and commercial species of Nicotiana.

\section{ACKNOWLEDGMENTS}

This research was supported in part by grants from the Tobacco and Health Research Institute (THRI) at the University of Kentucky and R. J.
Reynolds Company. E. P. Heist was the recipient of a Jeffrey Fellowship from the University of Kentucky. This is publication 00-12-171 of the Kentucky Agricultural Experiment Station, published with the approval of the director. We thank G. Collins and R. Dinkins (University of Kentucky) for valuable advice concerning tissue culture techniques.

\section{LITERATURE CITED}

1. Aylor, D. E., Taylor, G. S., and Raynor, G. S. 1982. Long-range transport of tobacco blue mold spores. Agric. Meteorol. 27:217-232.

2. Clayton, E. 1945. Resistance of tobacco blue mold (Peronospora tabacina). J. Agric. Res. 70:79-87.

3. Davis, J. M., and Main, C. E. 1986. Applying atmospheric trajectory analysis to problems in epidemiology. Plant Dis. 70:490-497.

4. Heist, E. P., Nesmith, W. C., and Schardl, C. L. 2001. Cocultures of Peronospora tabacina and Nicotiana species to study host-pathogen interactions. Phytopathology 91:1224-1230.

5. Hill, A. V. 1966. Physiologic specialization in Peronospora tabacina Adam in Australia. Bulletin D'Information Du CORESTA 1:7-15.

6. Lucas, G. B. 1975. Black shank. Pages 115-141 in: Diseases of Tobacco. Biological Consulting Associates, Raleigh, NC.

7. Lucas, G. B. 1975. Damping-off. Pages 161-172 in: Diseases of Tobacco. Biological Consulting Associates, Raleigh, NC.

8. Lucas, G. B. 1975. Blue mold. Pages 235-266 in: Diseases of Tobacco. Biological Consulting Associates, Raleigh, NC.

9. Lucas, G. B. 1980. The war against blue mold. Science 210:147-153.

10. Milholland, R. D., Papadopoulou, J., and Daykin, M. 1980. Histopathology of Peronospora tabacina in systemically infected burley tobacco. Phytopathology 71:73-76.

11. Murashige, T., and Skoog, F. 1962. A revised medium for rapid growth and bioassays with tobacco tissue culture. Physiol. Plant 15:473497.

12. Nesmith, W. C. 1984. The North American blue mold warning system. Plant Dis. 68:933-936.

13. Novotelnova, N. S. 1966. Downy mildew of sunflower. Nauka Acad. Sci. USSR, Moscow.

14. Reuveni, M., Nesmith, W. C., and Siegel, M. R. 1986. Symptom development and disease severity in Nicotiana tabacum and $N$. repanda caused by Peronospora tabacina. Plant Dis. 70:727-729.

15. Reuveni, M., Nesmith, W. C., Siegel, M. R., and Keeny, T. M. 1988. Virulence of an endemic isolate of Peronospora tabacina from Texas to Nicotiana tabacum and N. repanda. Plant Dis. 72:1024-1027.

16. Reuveni, M., Tuzun, S., Cole, J. S., Siegel, M. R., and Ku•, J. 1986. The effects of plant age and leaf position on the susceptibility of tobacco to blue mold caused by Peronospora tabacina. Phytopathology 76 : 455-458.

17. Shipton, W. A., and Brown, J. F. 1962. A whole-leaf clearing and staining technique to demonstrate host-pathogen relationships of wheat stem rust. Phytopathology 52:1313.

18. Stanghellini, M. E., Adaskaveg, J. E., and Rasmussen, S. L. 1990. Pathogenesis of Plasmopara lactucae-radicis, a systemic root pathogen of cultivated lettuce. Plant Dis. 74:173-178.

19. Tuzun, S., and $\mathrm{Ku} \bullet$, J. 1985. A modified technique for inducing systemic resistance to blue mold and increasing growth in tobacco. Phytopathology 75:1127-1129.

20. Tuzun, S., Nesmith, W., Ferriss, R. S., and Ku•, J. 1986. Effects of stem injections with Peronospora tabacina on growth of tobacco and protection against blue mold in the field. Phytopathology 76:938941.

21. Wolf, F. A. 1947. Tobacco downy mildew, endemic to Texas and Mexico. Phytopathology 37:721-729.

22. Wolf, F. A., Dixon, L. F., McLean, R., and Darkis, F. R. 1934. Downy mildew of tobacco. Phytopathology 24:337-363. 